\title{
Lyapunov Exponents of Symmetric Attractors
}

\author{
Philip J Aston Ian Melbourne \\ Department of Mathematics \& Statistics \\ University of Surrey \\ Guildford GU2 7XH, UK
}

15 February, 2006

\begin{abstract}
The Lyapunov exponents of symmetric attractors can be forced to be multiple by "instantaneous symmetries" which fix the attractor pointwise. In this paper, we show that "symmetries on average" which fix the attractor as a set may lead to further multiplicities. This work is motivated by, and provides an explanation for, numerical computations by Aston \& Laing of Lyapunov exponents for the complex Ginzburg-Landau equation.
\end{abstract}

\section{Introduction}

In dynamical systems with symmetry, the existence of symmetric but chaotic dynamics is now well-established, see [7, 8, 10] for numerical examples, [13] for experimental verifications, and $[2,11]$ for rigorous existence results. Whereas for sufficiently smooth systems without symmetry one would expect that the Lyapunov exponents of chaotic attractors are distinct, it was shown by Aston \& Dellnitz [3] that the presence of symmetries may force the Lyapunov exponents to be multiple.

It is necessary to distinguish between two different types of symmetry associated with a symmetric attractor: instantaneous symmetries that fix the attractor pointwise, and symmetries on average that fix the attractor as a set [16]. The terminology is explained as follows. In a numerical simulation or a numerical experiment starting from a single initial condition, the instantaneous symmetry is the symmetry of the data recorded at any particular moment in time, whereas the symmetry on average is a property of the time-average of the data. Precise definitions are given in Section 2.

The aforementioned results of [3] concern the effects of instantaneous symmetry. At first sight, symmetry on average does not seem to have a significant effect on 
multiplicities of Lyapunov exponents; in particular in the absence of instantaneous symmetry, we do not expect that any constraints arise from the presence of average symmetries. Perhaps surprisingly, the coexistence of instantaneous and average symmetries impose constraints beyond those imposed by the instantaneous symmetries alone. It is the purpose of this paper to describe these constraints.

We note that related results have been obtained previously by Ashwin \& Breakspear [1]. Generally, symmetry forces the existence of dynamically-invariant subspaces. An attractor with nontrivial instantaneous symmetry may lie in a number of flowinvariant subspaces, and the existence of symmetries on average that permute these subspaces may lead to multiple Lyapunov exponents [1]. Our results do not make use of flow-invariant subspaces. Indeed, an example in Section 3 shows that our results are more general than those of [1] and it seems likely that our results are sharp. (We do not attempt to prove sharpness; even in the nonsymmetric context, definitive results on the simplicity of Lyapunov exponents are not yet available).

This work is motivated by numerical results of Aston \& Laing [5], where Lyapunov exponents were computed for the Complex Ginzburg Landau (CGL) equation. It was observed that two of the Lyapunov exponents were almost identical, and that this did not seem to be accounted for by the instantaneous symmetries. We now reconsider this example and show that this effect is due to symmetry on average.

In Section 2, the theory regarding Lyapunov exponents taking into account the symmetry on average of the attractor is developed. Illustrative examples are given in Section 3, and the example of multiple Lyapunov exponents in the Complex Ginzburg Landau equation is considered in more detail in Section 4.

\section{Lyapunov Exponents and Symmetry}

In this section, we describe the restrictions that instantaneous and average symmetry places on Lyapunov exponents. In Subsection 2.1, we recall the notions of instantaneous symmetry and symmetry on average for equivariant dynamical systems. In Subsection 2.2, we discuss the implications of the isotypic decomposition for the eigenvalues of a commuting linear map. In Subsection 2.3, we rederive (and slightly improve) results of [3] concerning Lyapunov exponents in the presence of instantaneous symmetry, and we obtain new results in the case of symmetry on average.

The results in this section are stated for maps but all the results also hold for ODE's and PDE's with the usual technical assumptions. 


\subsection{Symmetric dynamics}

Let $\Gamma \subset \mathbf{O}(n)$ be a compact Lie group acting orthogonally on $\mathbb{R}^{n}$. A map $f: \mathbb{R}^{n} \rightarrow \mathbb{R}^{n}$ is $\Gamma$-equivariant if $f(\gamma x)=\gamma f(x)$ for all $\gamma \in \Gamma, x \in \mathbb{R}^{n}$. Let $A \subset \mathbb{R}^{n}$ be a compact dynamically-invariant set (so $f(A)=A$ ). Following [16], we associate two symmetry groups to the set $A$ :

$$
\Delta=\{\gamma \in \Gamma: \gamma x=x \text { for all } x \in A\}, \quad \Sigma=\{\gamma \in \Gamma: \gamma A=A\} .
$$

Elements in $\Delta$ fix $A$ pointwise and can be interpreted as "instantaneous symmetry" (visible at any step of the iteration process). The subgroup $\Sigma$ has the interpretation of "symmetry on average" and consists of elements that fix $A$ as a set. We note that $\Sigma$ and $\Delta$ are closed subgroups of $\Gamma$ and that $\Delta$ is a normal subgroup of $\Sigma$.

Define the fixed-point subspace Fix $\Delta=\left\{x \in \mathbb{R}^{n}: \delta x=x\right.$ for all $\left.\delta \in \Delta\right\}$. Then Fix $\Delta$ is a dynamically-invariant subspace of $\mathbb{R}^{n}$ (i.e. $f(\operatorname{Fix} \Delta) \subset$ Fix $\Delta$ ). Note that $A \subset \operatorname{Fix} \Delta$.

\subsection{Commuting matrices and isotypic decompositions}

When $A=x_{0}$ is a fixed point, the subgroups $\Sigma$ and $\Delta$ coincide and are called the isotropy subgroup $\Delta$ of $x_{0}$. It is easily verified that the linearisation $(d f)_{x_{0}}$ commutes with elements $\delta \in \Delta$ and hence inherits the structure associated with such a commuting matrix. More precisely, $(d f)_{x_{0}} \in \operatorname{Hom}_{\Delta}\left(\mathbb{R}^{n}\right)$ where $\operatorname{Hom}_{\Delta}\left(\mathbb{R}^{n}\right)$ is the vector space of linear maps $L: \mathbb{R}^{n} \rightarrow \mathbb{R}^{n}$ satisfying $L \delta=\delta L$ for all $\delta \in \Delta$. More generally, if $A \subset \mathbb{R}^{n}$ is a compact invariant set with instantaneous symmetry $\Delta$, then $(d f)_{x} \in \operatorname{Hom}_{\Delta}\left(\mathbb{R}^{n}\right)$ for each $x \in A$.

The restrictions on the eigenvalues of $L \in \operatorname{Hom}_{\Delta}\left(\mathbb{R}^{n}\right)$ can be determined using representation-theoretic notions. Moreover, the computation of eigenvalues is greatly facilitated, see for example [12]. We recall these ideas now.

Recall that a subspace $V \subset \mathbb{R}^{n}$ is $\Delta$-invariant if $\delta V=V$ for all $\delta \in \Delta$. An invariant subspace $V$ is irreducible if $V$ contains no proper invariant subspaces. It follows from compactness of $\Delta$ that $\mathbb{R}^{n}$ can be written as a direct sum of irreducible subspaces.

Two $\Delta$-irreducible subspaces $V, W$ are $\Delta$-isomorphic if there is a nonsingular linear map $A: V \rightarrow W$ such that $A \delta=\delta A$ for all $\delta \in \Delta$. Write

$$
\mathbb{R}^{n}=W_{1} \oplus \cdots \oplus W_{\ell}
$$

where each $W_{j}$ is a direct sum of isomorphic copies of a single irreducible subspace, and distinct $W_{j}$ 's contain nonisomorphic irreducible subspaces. The decomposition (2.1) is unique up to the order of the summands, and is called the isotypic decomposition. The subspaces $W_{j}$ are called the isotypic components. Note that we may take $W_{1}=$ Fix $\Delta$. 
We recall without proof the following elementary result [12].

Proposition 2.1 Let $L \in \operatorname{Hom}_{\Delta}\left(\mathbb{R}^{n}\right)$ and suppose that $W$ is an isotypic component. Then $L W \subset W$.

It follows that every $L \in \operatorname{Hom}_{\Delta}\left(\mathbb{R}^{n}\right)$ has the block-diagonalisation

$$
L=\left.\left.L\right|_{W_{1}} \oplus \cdots \oplus L\right|_{W_{\ell}} .
$$

In particular, the eigenvalues of $L$ can be computed one isotypic component at a time.

There are restrictions on the eigenvalues of $L$ within each isotypic component. In this paper, we are interested in positive-definite matrices, so we restrict to real eigenvalues in the next result.

Proposition 2.2 Let $W=V \oplus \cdots \oplus V$ be an isotypic component corresponding to an irreducible representation $V$. Let $d=\operatorname{dim} V$. Suppose that $L \in \operatorname{Hom}_{\Delta}(W)$ is a commuting linear map with real eigenvalues. Then the eigenvalues of $\left.L\right|_{W}$ have multiplicity at least $d$. In general, the multiplicity is precisely $d$.

Proof This follows from standard results stated explicitly for example in [15, Theorem 1.3, Proposition 2.1]. We sketch the details for completeness. Restricting to $V$, we have that $\operatorname{Hom}_{\Delta}(V)$ is a division ring $\mathcal{D}$ isomorphic to $\mathbb{R}, \mathbb{C}$ or $\mathbb{H}$. Hence $\operatorname{Hom}_{\Delta}(W)$ is isomorphic to the space of $k \times k$ matrices with entries in $\mathcal{D}$. Passing to Jordan normal form over $\mathcal{D}$, the eigenvalues of $L$ are given up to multiplicities by the eigenvalues of $L^{\prime}=d_{1} \oplus \cdots \oplus d_{k}$ where $d_{1}, \ldots, d_{k} \in \mathcal{D}$. By the reality condition on the eigenvalues, $L^{\prime}$ has $k$ real eigenvalues of multiplicity $\operatorname{dim} \mathcal{D}$. Hence $L$ has $k$ real eigenvalues of multiplicity $d$. It is clear from the proof that there are no further restrictions on the eigenvalues.

\section{$2.3 \quad$ Lyapunov exponents}

We recall Oseledec's Theorem (see [18] or [14, Theorem S.2.9]) on the existence of Lyapunov exponents. In the absence of symmetry, we suppose that $M$ is a compact manifold of dimension $n$ and that $f: M \rightarrow M$ is a $C^{1}$ diffeomorphism. Let $m$ be an ergodic measure.

Theorem 2.3 (Oseledec's Theorem [18]) There exist $k \leq n$ real numbers (Lyapunov exponents) $\lambda_{1}>\cdots>\lambda_{k}$ with multiplicities $n_{1}, \ldots, n_{k} \geq 1\left(n_{1}+\cdots+n_{k}=n\right)$ with the following property: 
There is a measurable splitting $T_{x} M=E_{x}^{1} \oplus \cdots \oplus E_{x}^{k}$ (defined for almost every $x$ with respect to $m$ ) with $\operatorname{dim} E_{x}^{j}=n_{j}$ and $(d f)_{x}\left(E_{x}^{j}\right)=E_{f(x)}^{j}$, such that

$$
\lim _{N \rightarrow \infty} \frac{1}{N} \log \left\|\left(d f^{N}\right)_{x} v\right\|=\lambda_{j} \quad \text { for all nonzero } v \in E_{x}^{j}
$$

for almost every $x$.

An alternative characterisation of the Lyapunov exponents is the following: The $n \times n$ matrix

$$
\Lambda_{x}=\lim _{N \rightarrow \infty}\left(\left(d f^{N}\right)_{x}^{T}\left(d f^{N}\right)_{x}\right)^{1 / 2 N}
$$

exists almost everywhere and has eigenvalues $e^{\lambda_{j}}$ with multiplicity $n_{j}, j=1, \ldots, n$. (Note that the exponents $\lambda_{j}$ and their multiplicities are constant on a set of full measure, but that the matrix $\Lambda_{x}$ depends on $x$. Similarly, the subspaces $E_{x}^{j}$ in Theorem 2.3 depend on $x$.)

Instantaneous symmetry Now we recall the results of [3] on the implications of instantaneous symmetry for Lyapunov exponents. Assume as before that $\Gamma \subset \mathbf{O}(n)$ is a compact Lie group and that $f: \mathbb{R}^{n} \rightarrow \mathbb{R}^{n}$ is a $\Gamma$-equivariant diffeomorphism. Suppose that $A \subset \mathbb{R}^{n}$ is a compact invariant set with instantaneous symmetry $\Delta$ and symmetry on average $\Sigma$. Let $m$ be an ergodic measure supported in $A$. (Since $m$ is compactly supported, the conclusions of Theorem 2.3 remain valid even though the ambient space is no longer a compact manifold.)

Lemma 2.4 If $x \in \mathbb{R}^{n}$ is such that the limit $\Lambda_{x}$ in (2.3) is defined, then $\Lambda_{\gamma x}$ exists for all $\gamma \in \Gamma$, and

$$
\Lambda_{\gamma x}=\gamma \Lambda_{x} \gamma^{-1}
$$

In particular, $\Lambda_{x} \in \operatorname{Hom}_{\Delta}\left(\mathbb{R}^{n}\right)$ for almost every $x \in A$.

Proof Fix $N \geq 1$. By equivariance, $f^{N}(\gamma x)=\gamma f^{N}(x)$. It follows from the chain rule that $\left(d f^{N}\right)_{\gamma x}=\gamma\left(d f^{N}\right)_{x} \gamma^{-1}$. Hence

$$
\left(d f^{N}\right)_{\gamma x}^{T}\left(d f^{N}\right)_{\gamma x}=\left(\gamma\left(d f^{N}\right)_{x} \gamma^{-1}\right)^{T} \gamma\left(d f^{N}\right)_{x} \gamma^{-1}=\gamma\left(d f^{N}\right)_{x}^{T}\left(d f^{N}\right)_{x} \gamma^{-1}
$$

where we have used the orthogonality of the action of $\Gamma$. The result follows.

By Lemma 2.4, we are in a position to use the results from Subsection 2.2. Corresponding to the isotypic decomposition (2.1) for the action of $\Delta$, we have

$$
\Lambda_{x}=\left.\left.\Lambda_{x}\right|_{W_{1}} \oplus \cdots \oplus \Lambda_{x}\right|_{W_{\ell}}
$$


It thus makes sense to speak of the Lyapunov exponents associated to each isotypic component $W=V \oplus \cdots \oplus V$. By Proposition 2.2, the Lyapunov exponents within $W$ have multiplicity at least $d$ where $d=\operatorname{dim} V$.

Remark 2.5 This result improves [3, Section 2.5(ii)] which claims only multiplicity $d / \operatorname{dim} \mathcal{D}$. (Here, $\mathcal{D} \cong \mathbb{R}, \mathbb{C}, \mathbb{H}$ is the division ring introduced in the proof of Proposition 2.2.) In the absolutely irreducible case $(\mathcal{D}=\mathbb{R})$, the results coincide.

Symmetry on average Since $\Delta$ is a normal subgroup of $\Sigma$, the elements of $\Sigma$ permute the $\Delta$-isotypic components. Write $W \sim W^{\prime}$ if there exists $\sigma \in \Sigma$ such that

$$
\sigma W=W^{\prime}
$$

This defines an equivalence relation on the isotypic components.

Theorem 2.6 If $W \sim W^{\prime}$, then the Lyapunov exponents within $W$ and $W^{\prime}$ coincide with the same multiplicities.

Proof Since $\left.\Lambda_{x}\right|_{W}$ is determined by the iterates of $\left.(d f)_{x}\right|_{W}$, it follows from Oseledec's multiplicative ergodic theorem for measurable cocycles [14, Theorem S.2.9] that the Lyapunov exponents in $W$ can be computed using almost any $x \in A$.

Now choose $\sigma \in \Sigma$ with $\sigma W=W^{\prime}$. For almost every $x$, the Lyapunov exponents in $W$ can be computed using $x$ or $\sigma x$. Similarly for $W^{\prime}$. By Lemma 2.4,

$$
\left.\Lambda_{\sigma x}\right|_{W^{\prime}}=\left.\left(\sigma \Lambda_{x} \sigma^{-1}\right)\right|_{\sigma W}=\sigma\left(\left.\Lambda_{x}\right|_{W}\right) \sigma^{-1} .
$$

Hence the Lyapunov exponents in the two isotypic components coincide.

\section{Theoretical Examples}

In this section, we give a number of examples to illustrate Theorem 2.6. In Subsection 3.1, we consider examples with dihedral symmetry, and indicate consequences for systems of coupled cells. In Subsection 3.2, we consider an example with octahedral symmetry where the symmetry on average forces a Lyapunov exponent of multiplicity three. In Subsection 3.3, we construct an example where the fixed-point subspace method of [1] fails to predict the correct multiplicities. 


\subsection{Dihedral symmetry}

Recall that $\mathbb{D}_{m}, m \geq 2$, is the group of order $2 m$ consisting of the symmetries of a regular $m$-gon (or a rectangle in the case $m=2$ ) consisting of $m$-fold rotations and $m$ reflections.

We consider the case where $\Delta=\mathbb{D}_{m}$ and $\Sigma=\mathbb{D}_{2 m}$. The group $\mathbb{D}_{2 m}$ is generated by a rotation $\rho$ and a reflection $\kappa$ satisfying

$$
\rho^{2 m}=I, \quad \kappa^{2}=I, \quad \kappa \rho=\rho^{-1} \kappa .
$$

The normal subgroup $\mathbb{D}_{m}$ is generated by $\rho^{2}$ and $\kappa$.

Suppose that $\Gamma \subset \mathbf{O}(n)$ acts orthogonally on $\mathbb{R}^{n}$ and contains the subgroup $\Sigma=\mathbb{D}_{2 m}$. Let $A \subset \mathbb{R}^{n}$ be a compact dynamically-invariant set for a $\Gamma$-equivariant dynamical system with instantaneous symmetry $\Delta=\mathbb{D}_{m}$ and symmetry on average $\Sigma=\mathbb{D}_{2 m}$.

Focusing first on the instantaneous symmetry, we recall that the irreducible representations of $\mathbb{D}_{m}$ are of dimension one and two. It follows from [3] (or Lemma 2.4) that the Lyapunov exponents lying in isotypic components $W \subset \mathbb{R}^{n}$ corresponding to the two-dimensional irreducible representations are of multiplicity two. These are the only restrictions from the instantaneous symmetry.

Now we consider the effects of the symmetry on average.

$m$ odd: Write $\Sigma=\Delta \oplus \mathbb{Z}_{2}$ where $\mathbb{Z}_{2}$ is generated by $\sigma=\rho^{m}$. (Note that $\rho^{m}$ corresponds to rotation by $180^{\circ}$ and hence commutes with every element of $\Delta$.) In particular, $\sigma: \mathbb{R}^{n} \rightarrow \mathbb{R}^{n}$ is a commuting linear map, $\sigma \in \operatorname{Hom}_{\Delta}\left(\mathbb{R}^{n}\right)$. Hence $\sigma W=$ $W$ for each isotypic component $W \subset \mathbb{R}^{n}$ and so $\sigma^{\prime} W=W$ for all $\sigma^{\prime} \in \Sigma-\Delta$. Consequently, there are no restrictions arising from the symmetries on average when $m$ is odd.

$m$ even: We consider the one-dimensional and two-dimensional irreducible representations separately. For $m$ even, there are precisely four one-dimensional irreducible representations given by

(i) $\kappa=I, \rho^{2}=I$.

(ii) $\kappa=-I, \rho^{2}=I$.

(iii) $\kappa=I, \rho^{2}=-I$.

(iv) $\kappa=-I, \rho^{2}=-I$.

We label the corresponding four isotypic components $W_{i}, i=1,2,3,4$.

For the components $W_{1}$ and $W_{2}$, the action of $\rho$ commutes with the action of $\Delta$, so by the same reasoning used in the case $m$ odd, we conclude that there are 
no restrictions. We claim however that $\rho$ permutes $W_{3}$ and $W_{4}$. Let $x \in W_{3}$ so in particular $\kappa x=x$. We must decide between the alternatives $\rho x \in W_{3}$ or $\rho x \in W_{4}$. Using (3.1), we compute that

$$
\kappa(\rho x)=\rho^{-1} \kappa x=\rho^{-1} x=\rho \rho^{-2} x=-\rho x .
$$

That is, $\kappa$ acts as $-I$ on $\rho x$ so that $\rho x \in W_{4}$ as required.

We conclude that the Lyapunov exponents in $W_{3}$ are identical to those in $W_{4}$; in particular they have multiplicity two.

Finally, let $W$ be an isotypic component corresponding to a two-dimensional irreducible representation $V$. We claim that $\sigma W=W$ for all $\sigma \in \Sigma-\Delta$, so that again there are no restrictions beyond the multiplicity two given by the instantaneous symmetry. There are a number of ways to verify this claim. A particularly simple method is to suppose for contradiction that $\sigma W=W^{\prime}$ where $W^{\prime} \neq W$. Then we can choose irreducible representations $V \subset W, V^{\prime} \subset W^{\prime}$ such that $\sigma V=V^{\prime}$. Note that $V \cap V^{\prime} \subset W \cap W^{\prime}=\{0\}$. Any $\Sigma$-irreducible subspace of $\mathbb{R}^{n}$ that contains $V$ must contain $V \oplus V^{\prime}$, and hence is at least 4-dimensional. This gives the required contradiction, since $\Sigma=\mathbb{D}_{2 m}$ has only one- and two-dimensional irreducible representations.

To summarise:

Proposition 3.1 Suppose that $A \subset \mathbb{R}^{n}$ is a compact invariant set with instantaneous symmetry $\Delta=\mathbb{D}_{m}$ and symmetry on average $\Sigma=\mathbb{D}_{2 m}$ generated by $\rho$ and $\kappa$ as above. Let $W_{1}=$ Fix $\Delta$ and let $W_{2}$ denote the isotypic component where $\rho^{2}$ acts trivially and $\kappa$ acts as $-I$.

(a) The overall restrictions due to symmetry are that the Lyapunov exponents corresponding to $W_{1}$ and $W_{2}$ are simple and the remaining Lyapunov exponents are double.

(b) The restrictions in (a) are due to the combined effects of $\Delta$ and $\Sigma$ for the two isotypic components ( $W_{3}$ and $W_{4}$ above) corresponding to the further onedimensional representations that are present when $m$ is even. The remaining restrictions are due to the effect of $\Delta$ alone.

Coupled cells Dihedral symmetry naturally arises in rings of identical coupled cells. The simplest case that fits the framework described above occurs when $m=2$ so that $\Delta=\mathbb{D}_{2}$ and $\Sigma=\mathbb{D}_{4}$ in a ring of eight cells. The overall symmetry group is $\Gamma=\mathbb{D}_{8}$ and the phase space is $\mathbb{R}^{n}=\mathbb{R}^{8 p}$ where $p$ is the dimension of the internal dynamics of each cell. Labelling the state of the $i^{\text {th }}$ cell by $x_{i} \in \mathbb{R}^{p}, i=1, \ldots, 8$, the 
action of $\Gamma$ is generated by

$$
\begin{aligned}
& \kappa\left(x_{1}, x_{2}, x_{3}, x_{4}, x_{5}, x_{6}, x_{7}, x_{8}\right)=\left(x_{8}, x_{7}, x_{6}, x_{5}, x_{4}, x_{3}, x_{2}, x_{1}\right), \\
& \gamma\left(x_{1}, x_{2}, x_{3}, x_{4}, x_{5}, x_{6}, x_{7}, x_{8}\right)=\left(x_{8}, x_{1}, x_{2}, x_{3}, x_{4}, x_{5}, x_{6}, x_{7}\right) .
\end{aligned}
$$

We let $\rho=\gamma^{2}$ so that $\Sigma$ is generated by $\rho$ and $\kappa$, and $\Delta$ is generated by $\rho^{2}$ and $\kappa$.

A subset $A$ with instantaneous symmetry $\Delta$ consists of points of the form

$$
\left(x_{1}, x_{2}, x_{3}, x_{4}, x_{5}, x_{6}, x_{7}, x_{8}\right)=(\alpha, \beta, \beta, \alpha, \alpha, \beta, \beta, \alpha) .
$$

A vector in the isotypic component $W_{3}$ then has the form

$$
\left(x_{1}, x_{2}, x_{3}, x_{4}, x_{5}, x_{6}, x_{7}, x_{8}\right)=(\alpha, \beta,-\beta,-\alpha,-\alpha,-\beta, \beta, \alpha) .
$$

Similarly, a vector in the isotypic component $W_{4}$ has the form

$$
\left(x_{1}, x_{2}, x_{3}, x_{4}, x_{5}, x_{6}, x_{7}, x_{8}\right)=(\beta, \alpha, \alpha, \beta,-\beta,-\alpha,-\alpha,-\beta) .
$$

It is easily seen in this case that $\rho W_{3}=W_{4}$ as anticipated.

\subsection{Octahedral symmetry}

Consider the 24 element octahedral group $\Sigma=\mathbb{O} \subset \mathbf{S O}(3)$ consisting of the rotations of a cube. There is a normal subgroup $\Delta=\mathbb{D}_{2}$ whose nontrivial elements are the $180^{\circ}$ rotations around axes connecting midpoints of faces. (In the standard coordinates, $\mathbb{D}_{2}$ consists of diagonal matrices with entries \pm 1 and determinant 1.)

As in Subsection 3.1, $\Delta$ has four one-dimensional irreducible representations with isotypic components $W_{1}, W_{2}, W_{3}, W_{4}$. These are the only isotypic components and there are no restrictions on the Lyapunov exponents from the instantaneous symmetry alone.

It is obvious on geometric grounds that the three nontrivial isotypic components $W_{2}, W_{3}, W_{4}$ are permuted by symmetries in $\Sigma-\Delta$. (A proof is obtained by considering the natural action of $\mathbb{O}$ as $3 \times 3$ rotations of $\mathbb{R}^{3}$. All three nontrivial representations of the subgroup $\mathbb{D}_{2}$ are present, and $\mathbb{O}$ acts irreducibly.) By Theorem 2.6, the corresponding Lyapunov exponents have multiplicity three.

Remark 3.2 Exactly the same considerations apply if $\mathbb{O}$ is replaced by any of the following subgroups of $\mathbf{O}(3)$ :

$$
\mathbb{O} \oplus \mathbb{Z}_{2}^{c}, \quad \mathbb{O}^{-}, \quad \mathbb{T} \oplus \mathbb{Z}_{2}^{c}, \quad \mathbb{T} .
$$

Here $\mathbb{Z}_{2}^{c}=\left\{ \pm I_{3}\right\}, \mathbb{T} \subset \mathbf{S O}(3)$ is the group of rotations of the tetrahedron, and $\mathbb{O}^{-}=\mathbb{T} \cup-I_{3} \cdot(\mathbb{O}-\mathbb{T})$.

Remark 3.3 We have the identifications $\mathbb{O} \cong \mathbb{O}^{-} \cong S_{4}$ (the symmetric group on 4 symbols) and $\mathbb{T} \cong A_{4}$ (the alternating group on 4 symbols), with $\mathbb{D}_{2}=$ $\{1,(12)(34),(13)(24),(14)(23)\}$. 


\subsection{An example with few fixed-point subspaces}

The following example shows that there are restrictions other than those that arise from fixed-point subspaces. So our treatment is more general than [1].

Take $\Sigma=\mathbb{Z}_{8} \rtimes \mathbb{Z}_{2}$ acting on $\mathbb{R}^{5} \cong \mathbb{C}^{2} \times \mathbb{C}^{2} \times \mathbb{R}$ as

$$
\begin{aligned}
& \rho\left(z_{1}, z_{2}, w\right)=\left(e^{\pi i / 4} z_{1}, e^{3 \pi i / 4} z_{2}, w\right), \\
& \tau\left(z_{1}, z_{2}, w\right)=\left(z_{2}, z_{1},-w\right) .
\end{aligned}
$$

Note that $\tau \rho \tau^{-1}=\rho^{3}$, so this indeed defines a semi-direct product.

Take $\Delta=\mathbb{Z}_{8}$ with fixed-point subspace $\left\{z_{1}=z_{2}=0\right\}$. The isotypic decomposition with respect to $\Delta$ is given by

$$
\{(0,0, w)\} \oplus\left\{\left(z_{1}, 0,0\right)\right\} \oplus\left\{\left(0, z_{2}, 0\right)\right\}
$$

where the first subspace is Fix $\Delta$. The results of [3] predict that the Lyapunov exponents in $\left\{\left(z_{1}, 0,0\right)\right\}$ are of multiplicity two, as are those in $\left\{\left(0, z_{2}, 0\right)\right\}$.

Taking into account the symmetry on average, we note that $\tau$ interchanges the second and third isotypic components in (3.2). Hence our results predict that the exponents in these subspaces are identical, and there is a Lyapunov exponent of multiplicity four in addition to the simple exponent in Fix $\Delta$. Moreover, it is easily verified that Fix $\Delta$ is not contained in any proper fixed-point subspaces, so this further restriction is not predicted by [1].

(The complete list of isotropy subgroups is $\Sigma, \Delta, \mathbb{Z}_{2}\left(\rho^{2} \tau\right), \mathbb{Z}_{2}(\tau)$ and $\mathbf{1}$ with fixed-point subspaces $\{0\},\{(0,0, w)\},\{(z,-i z, 0)\},\{(z, z, 0)\}$ and $\mathbb{R}^{5}$ respectively.)

\section{Multiple Lyapunov Exponents in the CGL Equation}

We now turn to the example of the Complex Ginzburg-Landau equation considered by Aston \& Laing [5] and present numerical results which indicate that the multiple Lyapunov exponents observed are due to the symmetry on average of the attractor, in agreement with the theory presented in Section 2.

The Complex Ginzburg-Landau equation in one space dimension is given by

$$
A_{t}=R A+(1+i \nu) A_{x x}-(1+i \mu) A|A|^{2}, \quad x \in[0,2 \pi),
$$

where $A \in \mathbb{C}, R, \nu, \mu \in \mathbb{R}$ and periodic boundary conditions are assumed. The symmetry group is $\Gamma=\mathbf{O}(2) \times S^{1}$ where $\mathbf{O}(2)$ consists of spatial translations and 
reflections acting as

$$
\begin{aligned}
r_{\alpha} A(x, t) & =A(x+\alpha, t), \quad \alpha \in[0,2 \pi) \\
s A(x, t) & =A(-x, t),
\end{aligned}
$$

and $S^{1}$ consists of rotations of the complex amplitude

$$
\theta A(x, t)=e^{i \theta} A(x, t), \quad \theta \in[0,2 \pi) .
$$

Solutions which have period $\pi$ and are even functions of $x$ are contained in the fixed point space Fix $\Delta$, where

$$
\Delta=\left\{I, r_{\pi}, s, s r_{\pi}\right\} \cong \mathbb{D}_{2} .
$$

We are interested in chaotic attractors with instantaneous symmetry $\Delta=\mathbb{D}_{2}$.

Remark 4.1 It follows from a general result of Melbourne \& Stewart [17] that chaotic attractors in systems with $\mathbf{O}(2) \times S^{1}$ have symmetry on average at least $S^{1}$ with probability one. Since $\Delta$ is a normal subgroup of $\Sigma$, the only possibilities for the symmetry on average are $\Sigma=\mathbb{D}_{2} \times S^{1}$ and $\Sigma=\mathbb{D}_{4} \times S^{1}$. Hence, effectively this problem is equivalent to the example considered in Subsection 3.1 with $m=2$.

The irreducible representations of $\Delta$ are all one-dimensional and the four isotypic components are given by

$$
\begin{array}{ll}
W_{1}=\left\{A \in X: s A=A, r_{\pi} A=A\right\}, & W_{2}=\left\{A \in X: s A=-A, r_{\pi} A=A\right\}, \\
W_{3}=\left\{A \in X: s A=A, r_{\pi} A=-A\right\}, & W_{4}=\left\{A \in X: s A=-A, r_{\pi} A=-A\right\} .
\end{array}
$$

We obtained numerical results using a pseudo-spectral method as described in [4] with $R=4, \mu=-4$ and $1.9 \leq \nu \leq 2.3$. The solution was left to settle down to the chaotic attractor for a transient time period of length 15 before computing the Lyapunov exponents for a time of 200. The dominant Lyapunov exponent $\lambda_{1}$ associated with $W_{1}=$ Fix $\Delta$, and the dominant Lyapunov exponents $\lambda_{3}$ and $\lambda_{4}$ associated with the isotypic components $W_{3}$ and $W_{4}$, are shown in Fig. 1 . The difference between $\lambda_{3}$ and $\lambda_{4}$ is shown in Fig. 2 and is generally quite small.

As described in Subsection 3.1, the isotypic components $W_{3}$ and $W_{4}$ are related by the relation $r_{\pi / 2} W_{3}=W_{4}$. If $\Sigma=\mathbb{D}_{4} \times S^{1}$, then by Theorem 2.6 the Lyapunov exponents in $W_{3}$ and $W_{4}$ coincide. It remains to verify, numerically at least, that the chaotic attractor has this symmetry on average.

To discuss symmetry on average, we consider the time average of $|A|$, defined by

$$
\widehat{|A|}(x)=\lim _{t \rightarrow \infty} \frac{1}{t} \int_{0}^{t}\left|A\left(x, t^{\prime}\right)\right| d t^{\prime} .
$$




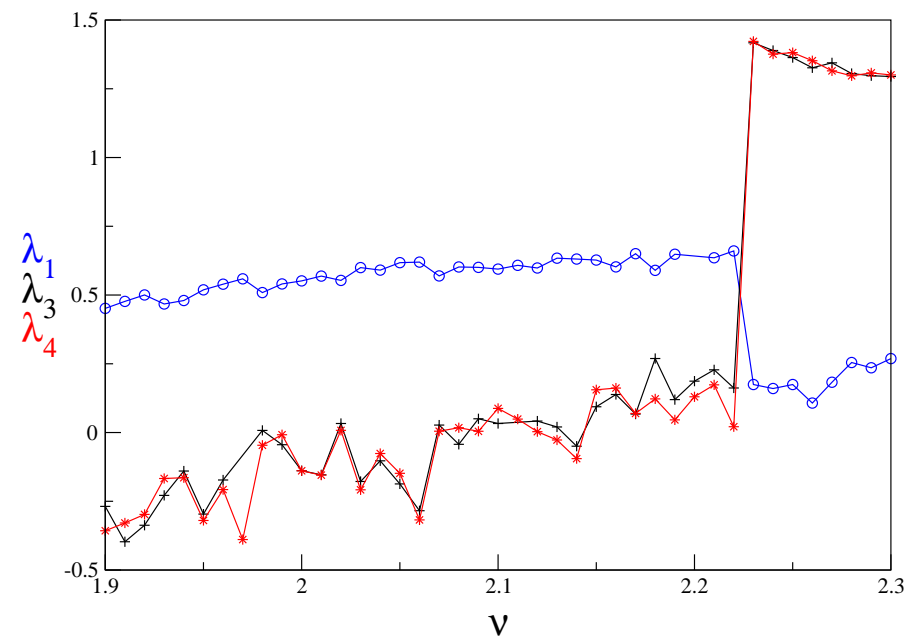

Figure 1: The dominant Lyapunov exponents associated with the isotypic components $W_{1}=$ Fix $\Delta$ (blue circle), $W_{3}$ (black plus) and $W_{4}$ (red star).

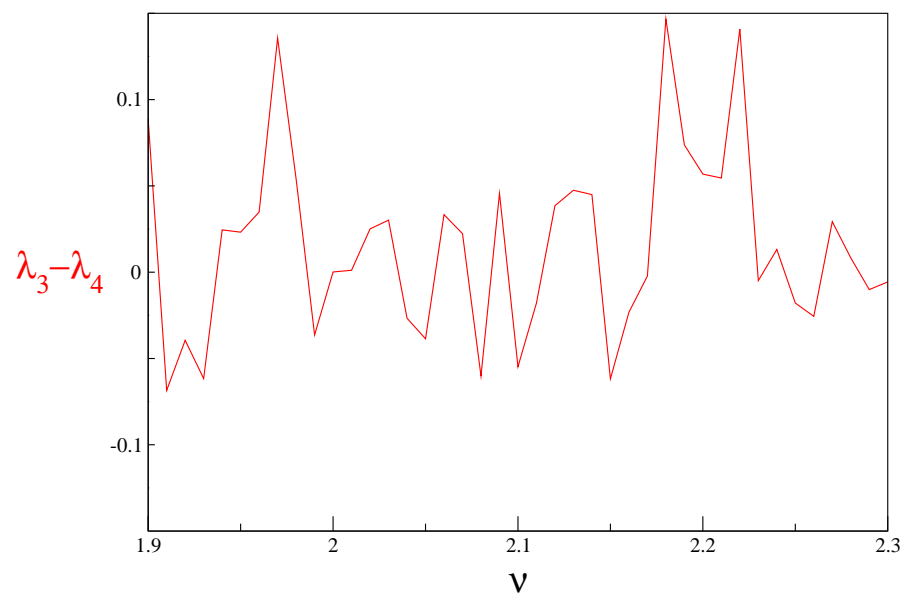

Figure 2: The difference between the Lyapunov exponents $\lambda_{3}$ and $\lambda_{4}$ shown in Fig. 1. 


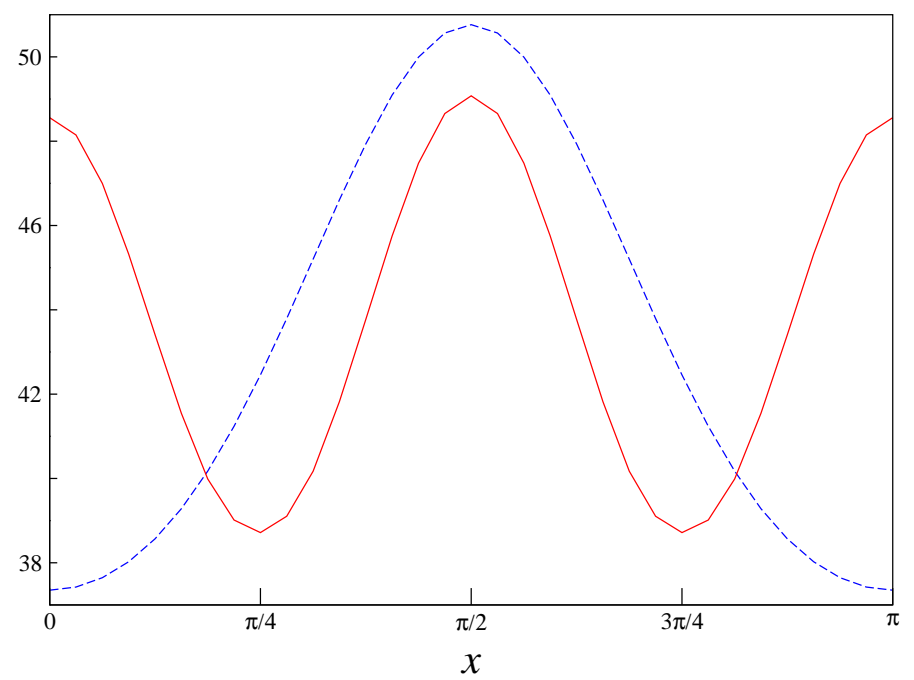

Figure 3: $|A(x, 200)|$ (blue dashed line), and $\widehat{|A|}(x)$ (red solid line) where the average is over a time period of 200. The parameter value is $\nu=2.3$.

This average, together with $|A|$ at one point in time are shown in Fig. 3, where the $\mathbb{D}_{2}$ symmetry of $|A|$ and the $\mathbb{D}_{4}$ symmetry of $\widehat{|A|}$ can be clearly seen. These results confirm that the symmetry on average of the attractor is $\Sigma=\mathbb{D}_{4} \times S^{1}$, as expected.

Remark 4.2 Note that the average $\widehat{A}(x)$ of $A$ fails to detect $\mathbb{D}_{4}$ symmetry on average. Typically $\Sigma$ contains the $S^{1}$ symmetry generated by the rotation $\theta$ (Remark 4.1), and automatically $\widehat{A}(x) \equiv 0$. Numerically, it was found that $\widehat{A}(x)$ is indeed (approximately) zero, in accordance with the prediction of [17].

We now define a scalar function to act as a detective [6, 9] for the symmetry on average. There are many possibilities, but we choose

$$
\delta_{0}=|\widehat{A}|(0)-\widehat{|A|}\left(\frac{\pi}{2}\right) \mid
$$

If $\Sigma=\mathbb{D}_{4} \times S^{1}$, then $\delta_{0}=0$ but if $\Sigma=\mathbb{D}_{2} \times S^{1}$, then generically $\delta_{0}>0$. For comparison, we also compute

$$
\delta_{1}=\left|\widehat{A A}(0)-\widehat{|A|}\left(\frac{\pi}{4}\right)\right|
$$

which is generically non-zero if $\Sigma=\mathbb{D}_{2} \times S^{1}$ or $\Sigma=\mathbb{D}_{4} \times S^{1}$. To remove the scale of the solution from these quantities, we normalise by

$$
\max _{x \in[0, \pi]} \widehat{|A|}(x)-\min _{x \in[0, \pi]} \widehat{|A|}(x)
$$




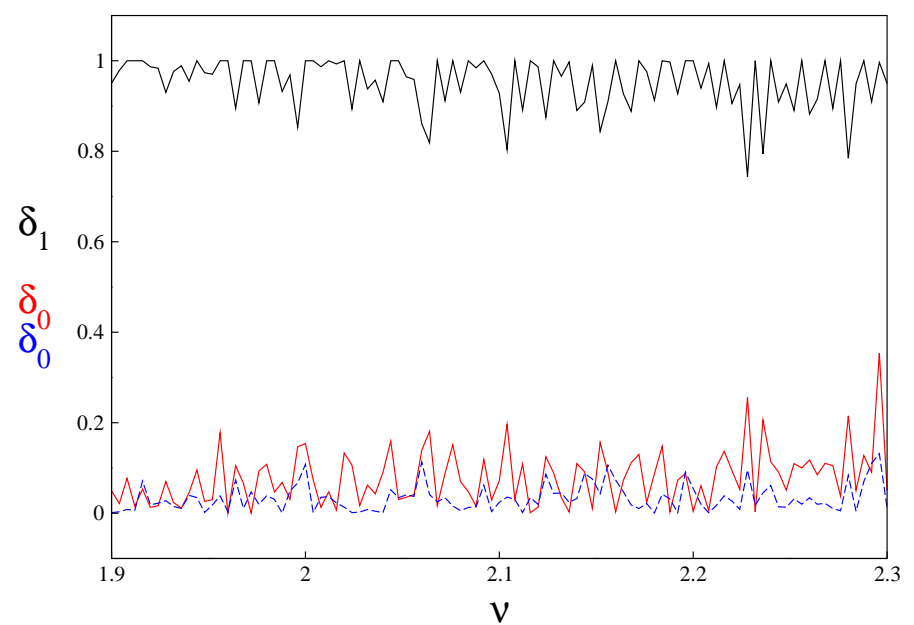

Figure 4: Symmetry detectives for $1.9 \leq \nu \leq 2.3$. The upper line is $\delta_{1}$. The two lower lines are $\delta_{0}$. The solid upper (black) and solid lower (red) lines are for an average over a time interval of 200 and the dashed lower line (blue) is for an average over a time interval of 1000 .

when computing $\delta_{0}$ and $\delta_{1}$.

The normalised quantities $\delta_{0}$ and $\delta_{1}$ computed over a time interval of 200 are shown in Fig. 4 for a range of values of $\nu$. As anticipated, $\delta_{0}$ is close to 0 whereas $\delta_{1}$ is of order 1. Fig. 4 shows also the improved values of $\delta_{0}$ after a time interval of 1000 .

The convergence towards zero of $\delta_{0}$ (normalised but without taking absolute values) is shown in Fig. 5 for the parameter value $\nu=2.3$.

These results indicate that the symmetry on average is indeed $\mathbb{D}_{4} \times S^{1}$ over the whole range of parameter values considered. Thus, by Theorem 2.6, the Lyapunov exponents associated with the isotypic components $W_{3}$ and $W_{4}$ are identical.

A bifurcation analysis of equilibria in equations with $\mathbb{D}_{2}$ symmetry shows that all three symmetry-breaking bifurcations associated with zero eigenvalues whose eigenvectors are in $W_{2}, W_{3}$ or $W_{4}$ give rise to a branch of solutions with $\mathbb{Z}_{2}$ symmetry, where the particular form of this symmetry is different in the three cases. However, in this chaotic case, since the two Lyapunov exponents associated with $W_{3}$ and $W_{4}$ are identical, a blowout bifurcation [19] would result in an attractor with no (pointwise) symmetry. An interesting question that we have not explored is how much symmetry on average the attractor will have after such a blowout bifurcation.

Acknowledgements The research of IM was supported in part by a Leverhulme Research Fellowship. 


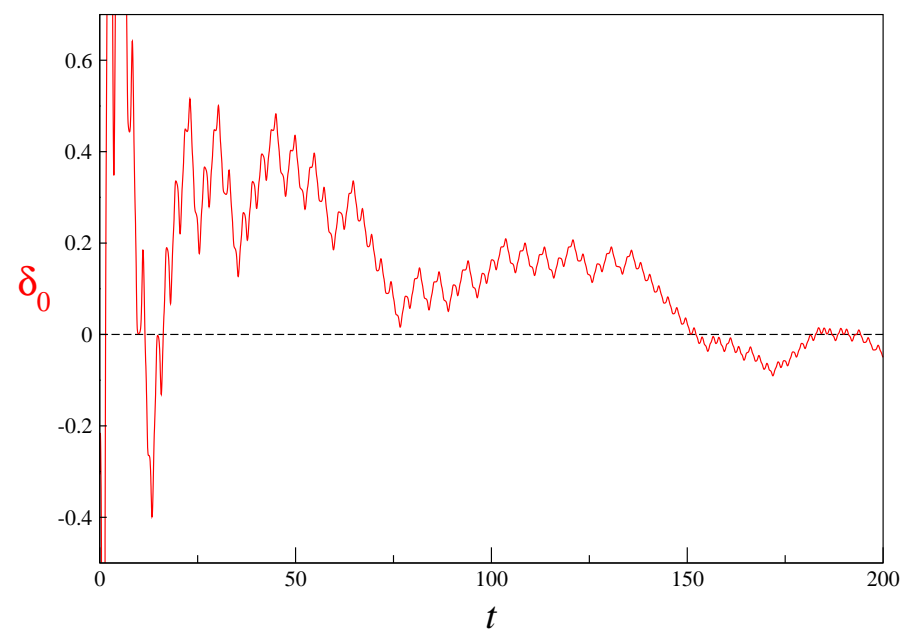

Figure 5: The symmetry detective $\delta_{0}$ against the time over which the average was taken, for $\nu=2.3$.

\section{References}

[1] P. Ashwin and M. Breakspear. Anisotropic properties of riddled basins. Phys. Lett. A 280 (2001) 139-145.

[2] P. Ashwin and I. Melbourne. Symmetry groups of attractors. Arch. Rational Mech. Anal. 126 (1994) 59-78.

[3] P. J. Aston and M. Dellnitz. Symmetry breaking bifurcations of chaotic attractors. Int. J. Bif. Chaos 5 (1995) 1643-1676.

[4] P. J. Aston and C. R. Laing. Symmetry and chaos in the complex GinzburgLandau equation I. Reflectional symmetries. Dyn. Stab. Sys. 14 (1999) 233-253.

[5] P. J. Aston and C. R. Laing. Symmetry and chaos in the complex GinzburgLandau equation II. Translation symmetries. Physica D 135 (2000) 79-97.

[6] E. Barany, M. Dellnitz, and M. Golubitsky. Detecting the symmetry of attractors. Physica D 67 (1993) 66-87.

[7] P. Chossat and M. Golubitsky. Iterates of maps with symmetry. SIAM J. Math. Anal. 19 (1988) 1259-1270.

[8] M. Dellnitz, M. Golubitsky and I. Melbourne. Mechanisms of symmetry creation. Bifurcation and Symmetry (E. Allgower et al., ed.), ISNM 104, Birkhäuser, Basel, 1992, pp. 99-109. 
[9] M. Dellnitz, M. Golubitsky, and M. Nicol. Symmetry of attractors and the Karhunen-Loève decomposition. Trends and Perspectives in Applied Mathematics (L. Sirovich, ed.), Appl. Math. Sci. 100, Springer, 1994, pp. 73-108.

[10] M. J. Field and M. Golubitsky. Symmetry in Chaos. Oxford Univ. Press, Oxford, 1992.

[11] M. J. Field, I. Melbourne and M. Nicol. Symmetric attractors for diffeomorphisms and flows. Proc. London Math. Soc. 72 (1996) 657-696.

[12] M. Golubitsky, I. N. Stewart and D. Schaeffer. Singularities and Groups in Bifurcation Theory, Vol. II, Appl. Math. Sci. 69, Springer, New York, 1988.

[13] B. J. Gluckman, P. Marcq, J. Bridge and J. P. Gollub. Time-averaging of chaotic spatiotemporal wave patterns. Phys. Rev. Lett. 71 (1993) 75-98.

[14] A. Katok and B. Hasselblatt. Introduction to the Modern Theory of Dynamical Systems. Encyclopedia of Math. and its Applications 54, Cambridge Univ. Press, Cambridge, 1995.

[15] I. Melbourne and M. Dellnitz. Normal forms for linear Hamiltonian vector fields commuting with the action of a compact Lie group. Math. Proc. Camb. Phil. Soc. 114 (1993) 235-268.

[16] I. Melbourne, M. Dellnitz and M. Golubitsky. Structure of symmetric attractors. Arch. Rational Mech. Anal. 123 (1993) 75-98.

[17] I. Melbourne and I. N. Stewart. Symmetric $\omega$-limit sets for smooth $\Gamma$-equivariant dynamical systems with $\Gamma^{0}$ abelian. Nonlinearity 10 (1997) 1551-1567.

[18] V. I. Oseledec. A multiplicative ergodic theorem. Lyapunov characteristic numbers for dynamical systems. Trudy Mosk. Mat. Obsc. 19 (1968) 197-231.

[19] E. Ott and J. C. Sommerer. Blowout bifurcations: the occurrence of riddled basins and on-off intermittency. Phys. Lett. A 188 (1994) 39-47. 